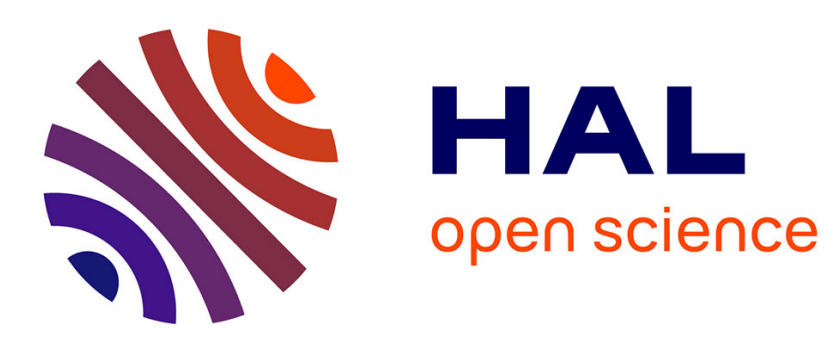

\title{
L'expression des TAM et la place des périphrases verbales dans trois langues
}

Richard Renault, Jacques François

\section{To cite this version:}

Richard Renault, Jacques François. L'expression des TAM et la place des périphrases verbales dans trois langues. Colloque: Les périphrases verbales, Jun 2003, Caen, France. 10.1075/lis.25.05ren . hal-00012686

\section{HAL Id: hal-00012686 https://hal.science/hal-00012686}

Submitted on 26 Oct 2005

HAL is a multi-disciplinary open access archive for the deposit and dissemination of scientific research documents, whether they are published or not. The documents may come from teaching and research institutions in France or abroad, or from public or private research centers.
L'archive ouverte pluridisciplinaire HAL, est destinée au dépôt et à la diffusion de documents scientifiques de niveau recherche, publiés ou non, émanant des établissements d'enseignement et de recherche français ou étrangers, des laboratoires publics ou privés. 


\title{
L'EXPRESSION DES TAM ET LA PLACE DES PÉRIPHRASES VERBALES DANS TROIS LANGUES
}

\author{
RICHARD RENAULT ET JACQUES FRANÇOIS \\ CRISCO, FRE 2805 du CNRS, Université De Caen
}

\section{Introduction}

Concernant particulièrement le Français et plus généralement les langues proches - en tout premier lieu les langues romanes - la notion de périphrase verbale a été forgée initialement pour "rendre compte des correspondances entre le latin, langue fusionnante et synthétique qui rend plusieurs notions par la même forme, et le français, langue analytique, qui tend à exprimer chacune d'elles par des mots graphiques différents, relativement autonomes les uns des autres, et parfois séparables"1. A ce titre [j'] ai fait, comme équivalent analytique du parfait latin feci est une périphrase verbale. Toutefois il n'est pas d'usage de parler en grammaire française de 'conjugaison périphrastique' à propos des temps composés et des formes de voix passive, c'est-à-dire de formes constituées à partir d'un auxiliaire régissant un participe passé (je suis arrivé ; je suis licencié). La Grammaire d'aujourd'hui ${ }^{2}$ précise que la notion s'applique traditionnellement en français "aux groupements constitués par l'auxiliaire aller, les aspectuels, les modaux et les semi-auxiliaires diathétiques avec le verbe à l'infinitif auquel ils sont liés" et rappelle que "les temps composés sont originellement des périphrases verbales". Il ne le sont donc plus, ce qui signifie qu'ils sont progressivement entrés dans le système verbal par grammaticalisation, c'est-à-dire par un effet de sclérose. L'opposition mentionnée plus haut entre l'expression synthétique lat. feci et l'expression analytique fr. $j$ 'ai fait n'est donc plus valide pour le français moderne et l'on peut opposer l'expression composée, j'ai fait à l'expression périphrastique je viens de faire tout comme l'expression synthétique je ferai (résultant historiquement d'une périphrase) et l'expression périphrastique je vais faire. En anglais, le système de flexion verbale a grammaticalisé le passif (be + participe passé) aussi bien que l'aspect accompli (have + participe passé) et que l'aspect progressif (be + participe présent) et le futur d'origine modale 
(will/shall + infinitif), de sorte que par exemple [ $I$ ] have been writing ou [it] had been written sont des expressions doublement composées qui, par grammaticalisation, ont perdu leur caractère analytique.

Notre propos sera ici d'interroger la notion de périphrase verbale d'un point de vue typologique en observant trois langues de structure très diverse, l'indonésien dont le système de flexion verbale est presque inexistant, le basque qui dispose d'un système de conjugaison traditionnellement appelée 'périphrastique' très riche incluant participe et infinitif et le finnois qui dispose d'une variété de formes nominales et participiales exerçant des fonctions diverses. Pour ce faire, nous partirons d'une typologie des différents moyens d'expression des morphèmes fonctionnels de la catégorie du verbe (temps, aspect et mode/modalité, dorénavant TAM) ${ }^{3}$ et nous préciserons la place des périphrases dans chacune des trois langues. La question que nous posons ce faisant est: "une notion initialement forgée pour rendre compte des équivalents analytiques d'une expression verbale synthétique (entre langues de tendance typologique différente, ex. lat. facitur vs. fr. c'est fait ${ }^{4}$ ou pour une même langue, ex. je partirai vs. je vais partir) est-elle un outil pertinent pour décrire des langues dont l'attirail de formes non finies diffère notablement de celui des langues (romanes) pour lesquelles elle a été conçue ?"

\section{Typologie des moyens d'expression des TAM}

La typologie que nous proposons reprend la typologie des moyens morphologiques de Mel'čuk ${ }^{5}$ réduite aux entités et aux modifications d'entités ${ }^{6}$, et étendue de manière à inclure les formes verbales complexes. En intégrant dans notre typologie les formes verbales complexes, nous obtenons un continuum dans lequel l'expression des TAM s'étend des formes verbales simples (obtenues par dérivation et/ou par flexion) aux formes verbales complexes dont le degré de cohésion entre constituants est de plus en plus lâche pour atteindre les structures coordonnées qui représentent une relation syntaxique libre ${ }^{7}$ :

(1) Continuum des formes verbales simples aux formes verbales complexes

dérivation $<$ flexion $<$ rection $<$ subordination $<$ cosubordination $<$ sérialisation $<$ coor dination

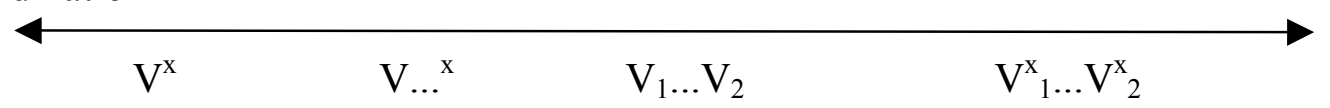

$\mathrm{V}=$ radical verbal, ${ }^{\mathrm{x}}=$ morphème de TAM 
Les principaux moyens d'expression des TAM dans ce continuum sont les suivants :

a. la flexion et la dérivation $\left(\mathrm{V}^{\mathrm{x}}\right)$ - Les morphèmes de $\mathrm{TAM}^{8}$ sont pris en compte dans le mot par un affixe ou par une modification de la forme du mot.

b. la rection $\left(\mathrm{V} . \mathrm{x}^{\mathrm{x}}\right)$ - Dans cette forme verbale complexe, une valeur de TAM est associée à un suffixe casuel de l'un des compléments du verbe. Dans les exemples finnois (2), le temps est induit par le cas morphologique de l'objet: L'aspect perfectif de l'accusatif (2a) couplé au morphème de présent correspond à un futur, tandis que l'aspect imperfectif du partitif correspond à un présent ${ }^{9}$ :

(2)
a. Luen
sanomalehden
lire+PRES +1 SG journal + GEN ${ }^{10}$
'Je lirai le journal'
b. Luen

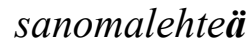
(perfectif $\Rightarrow$ futur)
lire+PRES+1SG journal+PART
'Je lis le journal'

c. la subordination $\left(\mathrm{V}_{1} \ldots \mathrm{V}_{2}\right)$ - Dans cette forme verbale complexe, le complément du verbe est une proposition. Les propositions complétives participiales ou nominales, en tant qu'objet nominal (la tête de la proposition reçoit le cas de l'objet), ont un statut intermédiaire entre rection et subordination.

d. la cosubordination $\left(\mathrm{V}_{1} \ldots \mathrm{V}_{2}\right)$ - Cette notion, empruntée à la grammaire de Van Valin et La Polla ${ }^{11}$ renvoie aux propositions dépendantes qui ne sont pas arguments du verbe. Cette relation de dépendance plus lâche que la complémentation vise ici les propositions adverbiales.

e. la sérialisation $\left(\mathrm{V}^{\mathrm{x}}{ }_{1} \ldots \mathrm{V}^{\mathrm{x}}{ }_{2}\right)$ - Dans les constructions sérielles ${ }^{12}$, les deux verbes partagent des arguments (ce qui les rapproche de la cosubordination) mais ne sont pas séparés par un enchâssement (ce qui les rapproche de la coordination).

f. la coordination $\left(\mathrm{V}^{\mathrm{x}}{ }_{1} \ldots \mathrm{V}^{\mathrm{x}}{ }_{2}\right)$ - Dans les structures coordonnées, les deux verbes sont fléchis indépendamment l'un de l'autre et appartiennent à deux propositions indépendantes. 


\section{La place des périphrases verbales dans les trois langues}

\subsection{L'indonésien}

Les parties du discours de l'indonésien ${ }^{13}$ se différencient difficilement en termes de codage, car l'indonésien n'a presque aucune flexion nominale ni verbale. Les verbes sont quasiment invariables, ne marquant aucune des catégories du temps, de l'aspect, de la personne, du nombre ou du mode. La voix passive est cependant marquée par le préfixe $d i$ - (passif dynamique) ou ter- (passif résultatif) et l'aspect répétitif est marqué par réduplication. Bien que verbes et adjectifs partagent cette propriété de quasi-invariabilité, ils se distinguent mutuellement en priorité à partir de critères d'affixation dérivationnelle, la dérivation lexicale présentant une régularité suffisante pour que les dictionnaires de langue soient organisés par familles lexicales. Ainsi, les verbes se reconnaissent à ce qu'ils présentent tous au moins une paire morphologique verbe nu vs. verbe dérivé par ber- (marque d'intransitivité), $m e$ - (marque de transitivité), $m e^{-*}-k a n$ (marque de causatif), etc. ${ }^{14}$. Le préfixe ter- dénote conceptuellement un aboutissement qui concerne dans le domaine verbal le résultat de l'action considérée du point de vue du patient, ex. Surabaya ter+letak di kuala Kali Mas (Surabaya est situé à l'embouchure de la rivière Mas) et dans le domaine adjectival le degré superlatif, par exemple baik (beau) > terbaik (le plus beau).

En raison du faible codage distinctif des parties du discours, le statut morphologique des constituants assurant le repérage temporel, aspectuel et modal est sujet à caution, comme le montre l'incertitude des terminologies employées par les grammairiens. La référence temporelle s'exprime par des syntagmes nominaux ou des mots simples classés par Mintz (1994 : 305-310) comme 'time adverbials' [abrégé TA], ex.

$\begin{array}{llll}\text { minggu lalu } & \text { la semaine dernière } & \text { dulu } & \text { avant/auparavant } \\ \text { minggu depan } & \text { la semaine prochaine } & \text { sekarang } & \text { maintenant } \\ \text { hari ini } & \text { aujourd'hui } & \text { kemarin } & \text { hier } \\ \text { malam ini } & \text { ce soir } & \text { besok } & \text { demain } \\ \text { pagi tadi } & \text { plus tôt ce matin (tantôt) } & \text { lusa } & \text { après-demain }\end{array}$

Les TA se disposent soit en fin de proposition soit en tête s'ils sont topicalisés, $\mathrm{ex}^{15}$ :

(3) Mereka belajar tadi vs Tadi mereka belajar

ils/elles étudi- avant avant ils/elles étudi-

'Ils/elles étudiaient avant" ${ }^{16} \underline{\text { vs }}$ 'avant ils/elles étudiaient' 


\section{(4) Mereka belajar nanti vs Nanti mereka belajar}

Ils/elles étudi- après après ils/elles étudi-

'Ils/elles étudieront plus tard' vs 'plus tard ils/elles étudieront'

Les visées aspectuelles et les modalités sont exprimées par des mots invariables disposés immédiatement avant le verbe. L'assignation catégorielle est très flottante: Labrousse (1997) les désigne tous comme des 'modalités', Grangé (2003) distingue les 'aspectual markers' des 'modal verbs' (sont cités bisa : pouvoir, dapat: être capable, boleh : avoir le droit, mau: vouloir, harus : être obligé ,(mais Mintz (1994) les considère comme des'auxiliaries'. Nous les noterons donc A/M (pour 'auxiliaire / modalité').

Labrousse donne (5) comme exemple de l'expression de l'obligation :

(5) Ibu harus pergi sekarang

Sujet $\mathrm{A} / \mathrm{M}<\bmod >\mathrm{V} \quad \mathrm{T}-\mathrm{Adv}$

Maman dev- part- maintenant [ou besok: demain]

'Maman va devoir partir maintenant'

tandis que Mintz illustre l'expression de la possibilité par (6) et celle de la visée aspectuelle accomplie par (7) :

$(6)^{17}$ Saya bisa tolong nenek sekarang

Sujet $\mathrm{A} / \mathrm{M}<\bmod >\mathrm{V} \quad$ Objet $\quad \mathrm{T}-\mathrm{Adv}$

moi pouv- aid- grand-mère maintenant

'Je peux aider grand-mère maintenant'

(7) Abang sudah pergi

Sujet A/M V

frère $\mathrm{ACCO}$ part-

'Mon frère est parti'

De un à trois $\mathrm{A} / \mathrm{M}$ peuvent être disposés entre le sujet et le verbe principal. Les $\mathrm{A} / \mathrm{M}$ de visée aspectuelle et de modalité peuvent se combiner dans l'ordre : (Négation) - $\mathrm{A} / \mathrm{M}_{<\mathrm{asp}>}-\mathrm{A} / \mathrm{M}_{<\bmod >}-\mathrm{V}$. Dans une communication spécialement consacrée à la combinatoire des $\mathrm{A} / \mathrm{M}$ en indonésien, Grangé (2003) illustre cette combinatoire par la structure (8) qui dispose la visée aspectuelle accomplie) pernah) dans la portée de la négation (tidak) et (9) où la négation (tidak) prend dans sa portée une visée prospective (akan) qui elle-même supporte une visée accomplie (pernah). 
(8) [sujet] tidak pernah sempat V

NEG ACCO avoir l'occasion V

'n'a jamais eu l'occasion de $I N F$ '

(9) [sujet] tidak akan pernah V

NEG PROSP ACCO V

'il n'arrivera jamais que [sujet $V]$ '

En conclusion, la notion de périphrase verbale ne se révèle pas opératoire pour une langue à codage distinctif limité des parties du discours, car le statut morphologique des marqueurs de référence temporelle, d'aspectualisation et de modalisation demeure incertain, entre adverbe et auxiliaire.

\subsection{Le basque $e^{18}$}

Le basque présente deux types de conjugaison dites respectivement 'synthétique' (c'est-à-dire faisant intervenir des morphèmes liés de flexion de part et d'autre du radical verbal) et 'périphrastique' (c'est-à-dire impliquant ce qu'il est convenu d'appeler un 'auxiliaire', bien que ce constituant soit dépourvu de tête lexicale). Nous ne nous intéresserons ici qu'au type périphrastique afin de chercher à élucider la combinatoire fonctionnelle entre la forme verbale fléchie et l'auxiliaire. Nous appuyant sur les paradigmes de conjugaison de la grammaire du basque labourdin de Lafitte (1962), nous n'illustrerons l'analyse qu'à la troisième personne du singulier.

Les formes périphrastiques sont constituées de deux composants : la forme verbale agglutinant le radical et un morphème aspectuel suffixé et la forme auxiliaire qui intègre des informations de cinq ordres différents. Commençons par l'auxiliaire. Chaque forme réalise un quintuplet de valeurs catégorielles de Personne, Nombre, Temps, Voix et Mode. Ainsi la forme d'auxiliaire $d u$ (dénotant la présence d'un sujet ergatif de $3^{\mathrm{e}}$ personne) se distingue minimalement de $d u t$ (sujet ergatif de $1^{\mathrm{e}}$ personne) et $d u z u$ (sujet ergatif de $2^{\mathrm{e}}$ personne), de dute et ditu (dénotant respectivement la présence d'un sujet ergatif ou d'un objet direct absolutif de nombre pluriel) par celle de nombre singulier, de da par la valeur de voix transitive vs. intransitive, de zuen par la valeur de présent vs. passé et de luke par la valeur modale de 'positif' vs. 'potentiel conjectural'.

Abstraction faite des deux premières catégories de la personne et du nombre, le jeu des oppositions de \{Voix x Temps x Mode\} délivre huit formes analysées dans la matrice ci-dessous : 
$(10)$

\begin{tabular}{llll}
\hline forme auxiliaire & \multicolumn{1}{c}{ Voix } & Temps & Mode \\
\hline$d u$ & transitive & présent & positif \\
\hline zuen & transitive & passé & positif \\
\hline$d a$ & intransitive & présent & positif \\
\hline zen & intransitive & passé & positif \\
\hline luke & transitive & présent & potentiel \\
\hline daiteke & transitive & passé & potentiel \\
\hline zatekeen & intransitive & présent & potentiel \\
\hline
\end{tabular}

ADD TITLE HERE

La sixième catégorie qui intervient dans la conjugaison périphrastique est l'aspect dont la marque se suffixe au radical verbal. Quatre valeurs de visée aspectuelle peuvent être marquées :

(i) la valeur imperfective (action en cours de déroulement) est marquée par le radical (forme de l'infinitif nominal) auquel se suffixent les deux marques du nominalisateur et $\mathrm{du}$ cas inessif: RAD+t(z)e+n . Métaphoriquement, l'action est envisagée d'un point de vue générique ${ }^{19}$ ou, en combinaison avec le verbe modal ari, exprimant l'idée d'action singulière, l'action est visée de l'intérieur ${ }^{20}$;

(ii) la valeur perfective par le participe à la forme casuelle de l'absolutif indéfini : RAD $+\boldsymbol{t u} / \boldsymbol{d} \boldsymbol{u}^{21}$

(iii) la valeur résultative par le participe à la forme casuelle de l'absolutif défini : RAD $+\boldsymbol{d u}+\boldsymbol{a}^{22}$

(iv) et enfin la valeur prospective par le participe à la forme casuelle du génitif en $-k o: \mathbf{R A D}+\boldsymbol{d u}+\boldsymbol{k o}$ 
(11)

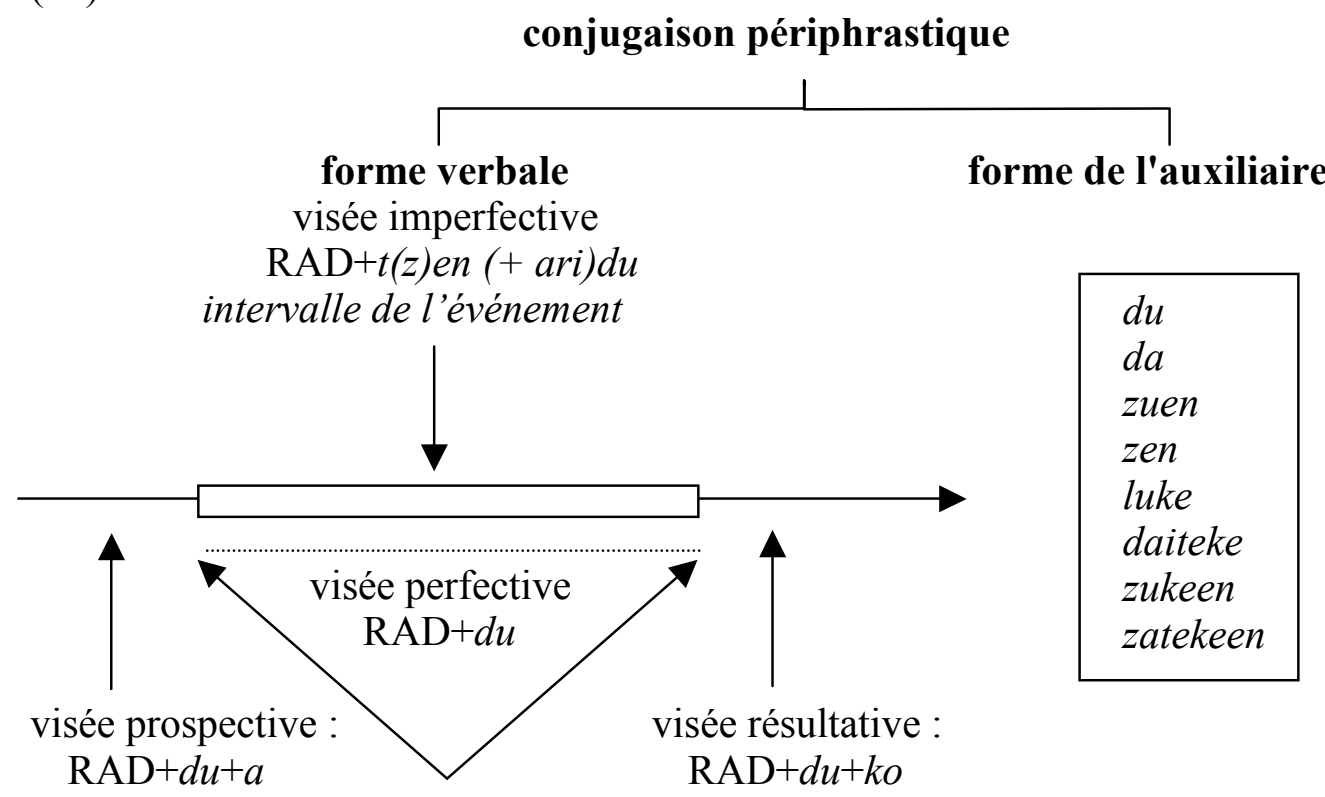

\section{ADD TITLE TO FIGURE}

En ce qui concerne la seule $3^{\mathrm{e}}$ personne du singulier, la combinatoire des 4 valeurs aspectuelles de la forme verbale et des 8 valeurs de l'auxiliaire résultant des trois oppositions binaires de voix, temps et mode délivre 32 combinaisons. A titre d'illustration, pour le radical verbal gal-neutre à l'égard de la voix et se traduisant par '(se) perdre' :

- galtzen $\boldsymbol{d u}$ s'analyse en $\{(\mathrm{se})$ perdre \& aspect : générique ou imperfectif $\}$ $\&\{$ voix : transitif \& temps : présent \& mode : positif\}, traduit par il perd (qch) et

- galdu zen en $\{(\mathrm{se})$ perdre \& aspect : perfectif\} \& \{voix : intransitif \& temps : passé \& mode : positif $\}$, traduit par il s'est perdu

Bottineau \& Roulland (2003) considèrent en outre que les formes de l'auxiliaire sont décomposables, les voyelles- $u$ et $-a$ exprimant respectivement la voix transitive (deux arguments respectivement à l'absolutif et à l'ergatif) et intransitive (un seul argument à l'absolutif). Cependant zuen s'oppose à zen et non zaen, zukeen à zatekeen et non zakeen et luke à daiteke et non lake. On est effectivement en présence d'une alternance de voix marquée par $-u$ vs $-a$, 
mais les conditions de combinabilité avec les deux autres valeurs de temps et mode demandent à être élucidées ${ }^{23}$.

En conclusion, la conjugaison 'périphrastique' du basque a la particularité de présenter une distribution originale des catégories fonctionnelles entre une forme verbale et une forme auxiliaire : du côté de la forme auxiliaire on trouve le marquage de la personne, du nombre, du temps, de la voix ${ }^{24}$ et du mode, du côté de la forme verbale celui de la visée aspectuelle. Cette prise en compte de la visée aspectuelle au plus près du radical verbal est directement liée à la valeur locative que celle-ci prend en basque, les marques d'aspect étant fondamentalement des marques de cas locatif dans l'argumentation de John Anderson (1973).

\subsection{Le finnois}

Toute la panoplie des moyens morphologiques proposée en (1) est disponible en finnois pour l'expression des valeurs de temps, d'aspect et de mode. Dans les exemples qui suivent, nous trouvons : a) des suffixes dérivationnels (12), b) des suffixes flexionnels (13), c) des mots appropriés (14), d) des formes verbales complexes (15) et (16), e) des constructions infinitives (17), et f) des constructions sérielles (18):

(12) a. -htA (suffixe sémelfactif) : katso- 'regarder', katsahta- 'jeter un coup d'œil' lepää- 'se reposer', levähtä' -faire une pause'

b. -i (suffixe duratif) : hyppä- 'sauter', hyppi- 'sauter'

(13) a. Tulin (passé)

venir+PRET $+1 \mathrm{SG}$

'Je suis venu(e)'

b. Hän sanoo minun tulleen eilen (antériorité)

il/elle dire+PRES $+3 \mathrm{SG}$ moi + GEN venir+PPA + ACC hier

'Il/elle dit que je suis venu(e) hier'

(14) a. temps (adverbes) : \{nyt 'maintenant', tänään 'aujourd'hui', huomenna 'demain'...

b. aspect (verbes de phase) : \{alkaa 'commencer', lopettaa 'finir', lakata 'cesser', jatkaa 'continuer'....

c. mode (verbes modaux): \{voida, saattaa 'pouvoir', täytyä, pitää 'falloir/devoir', tarvita 'avoir besoin de', sallia 'permettre'...\}
a. Olin kaatua (aspect avertif)

être+PRET+1SG tomber+INF1 ${ }^{25}$

'J'ai failli tomber' 
b. Olen lukemassa (aspect progressif)

être+PRES+1SG lire+INF3+INES

'Je suis en train de lire'

c. Olen kuolemaisillani (aspect imminent)

être+PRES+1SG mourir+INF3+PL+ILL+1SG

'Je suis sur le point de mourir'

d. Olen tavattavissa (modalité dynamique : possible, verbe transitif)

être+PRES+1SG rencontrer+PASS+PPR+PL+INES

'Je suis là (lit. *Je suis rencontrable)'

e. Olen nukkuvinani (modalité : simulation)

être+PRES+1SG dormir+PPR+ESS+1SG

'Je fais semblant de dormir'

f. Tulen lähtemään kotiin (temps futur)

venir+PRES+1SG partir+INF3+ILL maison+ILL

'Je partirai à la maison'

(16) a. Minun on

lähteminen (modalité : obligation)

moi+GEN être+PRES+3SG partir+INF4

'Je dois partir'

b. Minun on lähdettävä (modalité : obligation)

moi + GEN être+PRES+3SG partir+PASS + PPR

'Je dois partir'

c. Minun on pakko lähteä (modalité : obligation)

moi+GEN être+PRES+3SG obligation partir+INF1

'Je dois partir'

(17) a. Minun pitää lähteä (modalité : obligation)

moi+GEN devoir+PRES+3SG partir+INF1

'je dois partir'

b. Alkaa sataa (aspect inchoatif)

commencer+PRES +3 SG pleuvoir+INF1

'Il commence à pleuvoir'

c. Rupeaa satamaan (aspect inchoatif)

commencer+PRES+3SG pleuvoir+INF3+ILL

'Il commence à pleuvoir'

d. Voin tulla (modalité : possible)

pouvoir+PRES+1SG venir+INF3+ILL

'Je peux venir'

(18) a. Vesi nousee nousemistaan (aspect duratif/cumulatif)

eau monte+PRES+SG monter+INF4+PART+3

'L'eau ne cesse de monter' 


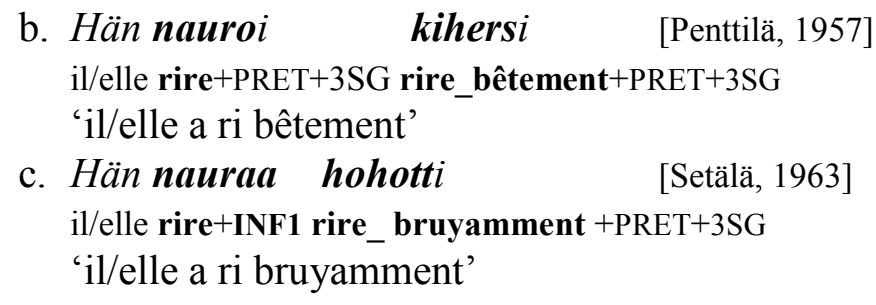

A. La dérivation lexicale est représentée dans nos exemples par les suffixes aspectuels sémelfactif (12a) et duratif (12b). Les deux exemples suivants illustrent l'opposition aspectuelle (ponctuel - duratif) ${ }^{26}$ :
a. Sitten mies hyppäsi
ikkunasta ja pum
puis homme sauter+PRET+3SG fenêtre+ELA et boum
'Puis l'homme a sauté de la fenêtre, et boom'
b. He tanssivat jahyppivät kuin kengurut
ils/elles danser+PRET+3PL et sauter+DURATIF+PRET+3PL comme kangourous 'Ils/elles ont dansé et sauté comme les kangourous'

B. Les exemples (13) illustrent respectivement les catégories flexionnelles du temps (prétérit) et de l'aspect (antériorité).

C. En (14), nous trouvons quelques exemples de mots appropriés pour chacune des trois catégories ; adverbes de temps (14a), verbes de phase (14b) et verbes modaux (14c).

D. Le dénominateur commun des formes verbales complexes (15) et (16) est la présence d'un lexème verbal sous la forme d'un participe ou d'un infinitif. L'auxiliaire de ces constructions est à une forme personnelle (15) ou impersonnelle (16). L'exemple (16c) se présente comme une transition entre les constructions avec auxiliaire et les phrases complexes avec verbe modal dans la mesure où l'infinitif, complément de nom, est tête d'une proposition infinitive avec sujet interne (au génitif) ${ }^{27}$.

E. On retrouve en (17) la distinction entre constructions personnelles et constructions impersonnelles dans les phrases complexes qui contiennent une proposition infinitive dépendante d'un verbe modal.

F. Les constructions sérielles de (18) reposent sur la présence de deux lexèmes verbaux contigus. La structure de l'exemple (18a) se caractérise par un redoublement du lexème verbal dont la première occurrence est une forme fléchie (temps et personne), tandis que la seconde est une forme nominale avec désinence personnelle. Le redoublement sert ici à l'expression de l'aspect duratif/cumulatif. La seconde structure de nature sérielle, peu usitée, est très différente : les deux lexèmes verbaux sont distincts et reliés par une relation 
d'hyponymie. Le premier verbe est soit fléchi (18b), soit à l'infinitif (18c). Ces constructions dites 'coloratives', de nature descriptive et emphatique, servent à introduire une nuance qui ne peut être rendue en français que par un adverbe.

\section{- Les périphrases du finnois}

Le critère que nous retenons pour délimiter les périphrases du finnois est le degré de nominalité de lexème verbal (opposition entre une forme nominale faible et forme nominale forte). Cette distinction s'inscrit dans un continuum qui va du verbe au nom en passant par les formes intermédiaires que sont les participes et les infinitifs. La place des participes et des infinitifs dans ce continuum est représentée par le schéma (20). Les propriétés pertinentes pour l'évaluation du degré de nominalité sont d'ordre syntagmatique et paradigmatique. Chacune des deux catégories se caractérise par la présence d'une ou plusieurs catégories fonctionnelles spécifiques (TAM pour les verbes, cas et nombre pour les noms ${ }^{28}$ ). La variation paradigmatique - ou l'absence de variation - dans chacune de ces catégories fonctionnelles est également un critère d'évaluation pour le degré de nominalité du lexème verbal. Dans les périphrases exprimant l'imminence et la simulation notamment (exemples 15c et 15e), la forme nominale est toujours au pluriel, sans qu'une variable de nombre soit engagée dans l'interprétation du procès.

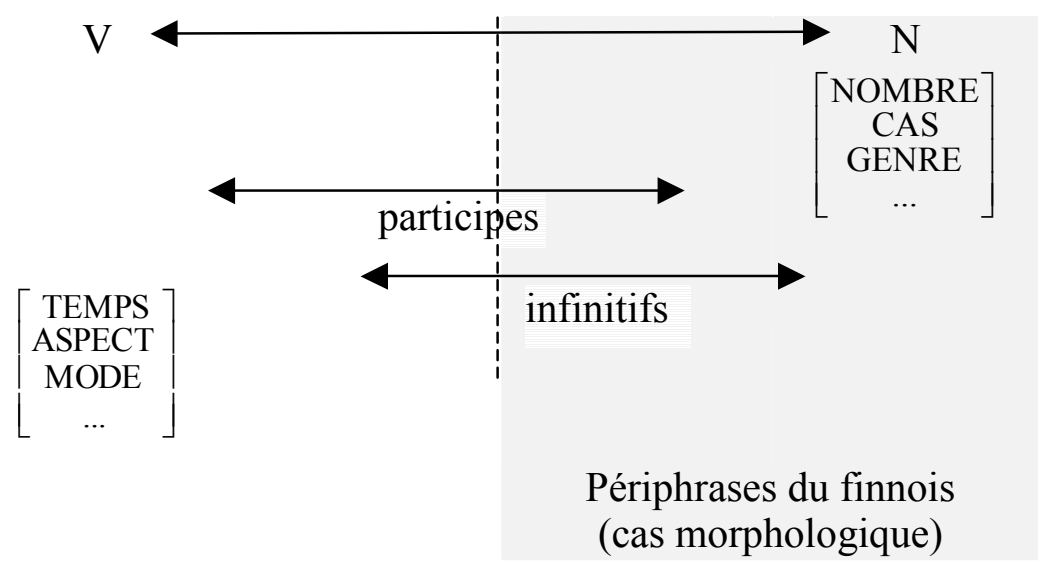

Participes, infinitifs et degré de nominalité

Comme le montre ce schéma, participes et infinitifs ont un degré de nominalité variable, les participes étant plus proches des verbes que les infinitifs. Quelques indications sur les propriétés extrêmes des participes et des infinitifs permettront de préciser ces deux points : Les participes sont spécifiés 
pour le temps ou l'aspect (propriétés verbales) et sont spécifiés également pour le nombre dans la construction auxiliaire/auxilié (c'est là leur seule propriété nominale). A l'autre bout de l'échelle, ils peuvent avoir en plus une spécification de cas. Ils partagent alors toutes les catégories fonctionnelles caractéristiques des noms. Les différents infinitifs des grammaires finnoises, correspondent également à des emplois syntaxiques plus ou moins nominaux. Le premier infinitif (exemples 15a, 16c, 17a, 17b et 18c) ) est un infinitif sans suffixe, qui n'admet à priori aucune marque casuelle. Il contient cependant en position finale une consonne virtuelle $\mathrm{e}^{29}$ qui s'interprète comme une marque casuelle de latif ${ }^{30}$. Ce premier infinitif, qui n'admet qu'un seul trait de cas morphologique (de nature abstraite) et dont l'objet obéit aux mêmes règles d'assignation casuelle que celles de l'objet d'un verbe conjugué est donc plus proche des verbes que des noms. A l'autre bout de l'échelle, on trouve le $4^{\mathrm{e}}$ infinitif (16a et 18a), qui n'est rien d'autre qu'un nom déverbal qui accepte les deux nombres et tous les suffixes casuels. Du fait de ces propriétés nominales très marquées, il est souvent assimilé à un suffixe dérivationnel.

Si l'on écarte les constructions verbales de type auxiliaire-auxilié dans lesquelles on observe une construction syntaxique qui met en jeu un morphème de temps (sur l'auxiliaire) et un morphème d'aspect (sur le participe), comme le montre le schéma (21a), les périphrases verbales du finnois se caractérisent comme la mise en relation d'un auxiliaire ou d'un pseudo-auxiliaire avec une forme nominale du verbe marquée pour un cas morphologique (21b).

\section{(21) a. olin lukenut (j'avais lu)

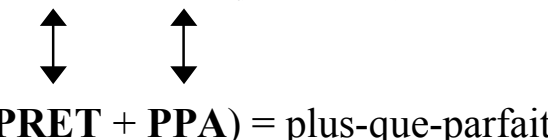 \\ b. olin lukemassa (j'étais en train de lire)}

Considérons maintenant quelques propriétés syntaxiques des périphrases finnoises qui confirment leur comportement nominal. Tout d'abord, une forme périphrastique comme celle du progressif prend place dans une même structure syntaxique que les compléments locatifs :
a. Olen lukemassa
être+PRES+1SG lire+INF3+INES
'Je suis en train de lire'
b. Olen saunassa
être+PRES+1SG sauna+INES
(aspect progressif)
'Je suis dans le sauna' 
Les deux phrases suivantes illustrent un autre rapprochement: d'un côté nous avons une périphrase impersonnelle avec sujet génitif et forme nominale du verbe au nominatif, et de l'autre, nous avons une phrase existentielle avec également un sujet génitif et un nom au nominatif:
a. Minun on
lähteminen
(modalité : obligation)
moi+GEN être+PRES+3SG partir+INF4
'Je dois partir'

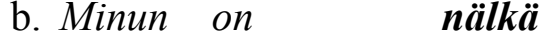
moi+GEN être+PRES+3SG faim
'J'ai faim'

La distribution de la négation et de l'auxiliaire permet d'isoler les périphrases parmi l'ensemble des formes verbales complexes. Le critère syntaxique qui permet d'écarter les constructions auxiliaire-auxilié est l'impossibilité d'avoir les deux auxiliaires (négation et ole- être) ${ }^{31}$, ensemble ou séparément, dans des propositions participiales et infinitives. On n'a pas en finnois d'équivalents directs de 'ne pas lire' ou 'ayant lu' ${ }^{32}$. Par contre, les périphrases qui contiennent le verbe ole- être sont possibles dans les constructions infinitives et participiales. Les exemples suivants, avec périphrases aspectuelles (progressif) et modales (possible) insérées dans une proposition infinitive ( $24 \mathrm{a}$ et $25 \mathrm{a}$ ) et participiale ( $24 \mathrm{~b}$ et $25 \mathrm{~b}$ ), illustrent ce point :

(24) Périphrase olla \# V+mA $+\mathbf{s s} \mathbf{A}$ (progressif)

a. Ilmalaivat saattavat [olla tekemässä paluuta] dirigeables peuvent être faire+INF3+INES retour Il se peut que les dirigeables soient sur le retour'

b. taidemaalari Antti Favènin [rapistumassa oleva] ateljee peintre A. F. délabrer+INF3+INES être+PPR atelier 'l'atelier du peintre Antti Favèn, qui est en train de se délabrer'

(25) Périphrase olla \# V+vA $+\mathbf{i}+\mathbf{s s} \mathbf{A}$ (possible)

a. Kaiken pitää [olla näkyvissä] tout doit être _visible $+\mathbf{P P R}+\mathbf{P L}+\mathbf{I N E S}$

'Tout doit être visible' 
b. sata [suomen kielellä saatavissa olevaa $] \quad k i r j a a$ cent finnois langue obtenir+PASS+PPR+PL+INES être+PPR+PART livre+PART 'cent livres disponibles en langue finnoise'

(lit : 'cent livres qu'on peut obtenir en langue finnoise')

Une périphrase verbale se présente donc en finnois comme un prédicat contenant une forme nominale du verbe marquée pour le cas .

\section{Conclusion}

Notre approche fondée sur l'expression des TAM permet d'écarter une caractérisation élémentaire des périphrases verbales en termes d'opposition entre formes synthétiques et analytiques, donc fondée uniquement sur le mot (notion particulièrement instable du point de vue typologique) au profit d'une caractérisation en termes de morphèmes de TAM.

La notion de périphrase est intimement liée en basque et en finnois aux propriétés nominales du lexème verbal. Dans ces deux langues, le lexème verbal contient pour le moins une marque de cas morphologique, qui, en corrélation avec un auxiliaire, induit une des valeurs de la catégorie TAM. Les périphrases verbales se présentent ainsi comme une stratégie d'expression nominale de catégories fonctionnelles verbales. La principale différence entre le basque et le finnois est que ces formes nominales sont complètement intégrées dans le système de conjugaison en basque (les formes nominales y sont plus nettement grammaticalisées), alors qu'en finnois les périphrases forment un ensemble de constructions intermédiaires entre les formes verbales simples et les phrases complexes. Quant à l'indonésien, l'application de cette notion de périphrase à l'expression des aspects et des modalités ne serait pertinente que si les critères d'identification des 'auxiliaires' étaient mieux établis.

En tout cas le modèle originel, à savoir les périphrases verbales des langues romanes constituées d'un verbe à fonction prédicative à un mode infinitif régi et d'un verbe auxiliaire recteur assurant une mise en perspective du procès, ne peut pas avoir de valeur définitoire en typologie dans la mesure où la morphologie verbale ne permet pas de distinguer un infinitif constitutif d'une périphrase d'un infinitif d'une construction syntaxique. La seule présence d'une forme infinitive ne suffit donc pas à caractériser une périphrase.

\section{Références}

Anderson, John M. 1973. An Essay Concerning Aspect : Some considerations of a General Character arising from the Abbé Darrigol's analysis of the Basque Verb. The Hague and Paris : Mouton. 
Bottineau, Didier \& Daniel Roulland. 2003. "La grammaticalisation de l'adresse en basque : formes allocutives, systématique, emploi et reconnaissance académique". Colloque International Pronoms de $2 e$ personne et formes d'adresse dans les langues d'Europe. Instituto Cervantes, Paris, 6-8 mars 2003.

Busang, Walter. 1991. "Verb serialization, grammaticalization and attractor positions in Chinese, Hmong, Vietnamese, Thai and Khmer". In Partizipation ed. by Seiler A \& Premper W., 509-562. Tübingen.

Bybee, Joan L. 1985. A study of the relation between meaning and form. Amsterdam/Philadelphia : John Benjamins Publishing Company.

Chomsky, Noam. 1995. The Minimalist Program. Cambridge MA : MIT Press.

Comrie, Bernard. 1976. Aspect. Amsterdam/Philadelphia : John Benjamins Publishing Compagny.

Comrie, Bernard. 1976. Tense. Amsterdam/Philadelphia : John Benjamins Publishing Compagny.

Creissels, Denis. 1994. Aperçu sur les structures phonologiques des langues négro-africaines. Grenoble : ellug.

Dahl, Östen. 2000. (ed.) Tense and aspect in the languages of Europe. Berlin/New York : Mouton de Gruyter.

Demirdache, Hamida et Uribe-Etxebarria, Myriam. 2002. "La grammaire des prédicats spatio-temporels, temps, aspect et adverbes de temps". Dans Temps et aspect ed. par Brenda Laca, 125-176. Paris : Presses Universitaires de Vincennes.

Etxebarne, Michel. 2002. Aditza, conjuguer le verbe basque (basque unifié). Bayonne : Elkar, Donostia.

Gosselin, Laurent. 1996. Sémantique de la temporalité en français. Un modèle calculatoire et cognitif. Louvain-la-neuve : Duculot.

------. 1999. "Le statut du temps et de l'aspect dans la structure modale de l'énoncé. Esquisse d'un modèle global". Syntaxe et Sémantique 2.57-80.

Grangé, Philippe. 2003. "Aspect and Modality in Indonesian : the case of telah, pernah, sempat". Nijmegen : ISMIL 7.

Guéron, Jacqueline \& Hoekstra Teun. 1988. "T-chains and the Constituent Structure of Auxiliaries". In Annali di Ca'Foscari ed. by Anna Cardinaletti, Gugliemo Cinque \& Guiliana Guisti. Venice : Annali di Ca'Foscari.

Guéron, Jacqueline. 1995. "Chaînes temporelles simples et structures auxiliaires". Dans Rencontres : études de syntaxe et de morphologie ed. par J. Guéron. université de Paris-X Nanterre.

Ikola, Osmo. 1978. Lauseenvastikeoppia. Tietolipas 76, SKS, Helsinki.

Kangasniemi, Heikki. 1992. Modal Expressions in Finnish. Studia Fennica Linguistica 2, SKS, Helsinki.

Karow, Otto. 1972. Vietnamesisch-deutsches Wörterbuch. Wiesbaden : Harrassowitz. 
Kaynes, Richard S. 1994. The Antisymmetry of Syntax. Cambridge MA : The MIT Press.

Klein, Wolfgang. 1995. "A Time-Relational Analysis of Russian Aspect". Language $71: 4.669-695$.

Klingler, Dominique. 2003. "Les relations de joncture dans RRG à travers l'exemple du connecteur affixal -te en japonais". Cahier du CRISCO 13.7798.

Labrousse, Pierre. 1997. Méthode d'indonésien. Langues \& Mondes, L'asiathèque, Paris.

Laca, Brenda. 2002. (ed.) Temps et aspect. Paris : Presses Universitaires de Vincennes.

Lafitte, Pierre. [1944, 1962] 1978. Grammaire basque (Navarro-labourdin littéraire). Bayonne : Elkar, Donostia.

Mel'čuk, Igor. 1993. Cours de morphologie générale. Les Presses de l'université de Montréal CNRS Editions.

-------. 1996. Cours de morphologie générale. Volume 3, troisième partie : moyens morphologiques. Les Presses de l'université de Montréal CNRS Editions.

Milner, Jean-Claude. 1989. Introduction à une science du langage. Paris : Edition du Seuil.

Mintz, Malcolm. 1994. A Student's Grammar of Malay and Indonesian. Singapour : EPB Publishers.

Palmer, Franck. 2001. (Second Edition) Mood and Modality. Cambridge MA : Cambridge University Press.

Penttilä, Aarni. 1957. Suomen Kielioppi. Porvoo : WSO

Pottier, Bernard. 1992. Sémantique générale. Paris : PUF.

Renault, Richard. 2004. "Cas locatifs et prédication". Syntaxe et sémantique 6.

Seiler, Hansjakob \& Premper, Walfried. (eds.) 1991. Partizipation. Das sprachliche Erfassen von Sachverhalten. Tübingen : Narr.

Setälä, Emil Nestor. 1963. Suomen kielen lauseoppi. Helsinki : Otava. Van Valin, Robert \& Randi La Polla. 1997. Syntax, Structure, Meaning and Function. Cambridge MA : Cambridge Universty Press.

\section{Notes}

${ }^{1} c f$. Dubois et al. (1973:367), article Périphrase.

${ }^{2} c f$. Arrivé et al. (1986:494), article Périphrase verbale.

${ }^{3}$ Nous laissons de côté la diathèse verbale.

${ }^{4} \mathrm{La}$ forme originellement analytique, puis composée par grammaticalisation, du passif présent en français, ex. [c']est fait résulte d'un déplacement de la référence temporelle à partir du passif parfait du latin factum est.

${ }^{5}$ Mel'čuk (1996). Cette typologie reconnait trois grands moyens morphologiques pour exprimer des éléments de contenu : les entités, les opérations et les relations d'ordre :

(i) Typologie des moyens morphologiques (Mel'čuk, 1996). 


$$
\begin{aligned}
& 1 \text { - entités }\left\{\begin{array}{l}
\text { segments } \\
\text { suprasegments }
\end{array}\right. \\
& 2 \text {-opérations }\left\{\begin{array}{l}
\text { Modifications } \\
\text { conversions }
\end{array}\right\}\left\{\begin{array}{l}
\text { segments } \\
\text { suprasegments }
\end{array}\right. \\
& 3 \text {-ordre }
\end{aligned}
$$

${ }^{6}$ Faute de place, nous ne pouvons pas présenter ici les raisons qui nous ont conduit à écarter les opérations de conversion et les relations d'ordre de notre typologie. En deux mots : les conversions peuvent être traitées plus efficacement dans le cadre d'une théorie des catégories qui admet le chevauchement des propriétés définitoires (catégories neutres, mixtes et duales) et les relations d'ordre entre constituants sont le plus souvent la manifestation d'une différence de position. Nous intégrons ici la distinction entre place (relation d'ordre) et position (relation de dominance) (Milner, 1989).

${ }^{7} \mathrm{Ce}$ continuum est à rapprocher de celui de la dimension universelle de la 'participation' développé par H. Seiler et l'équipe UNITYP (Recherche sur les universaux et typologie des langues, Université de Cologne) qui va des expressions holophrastiques, nominalisations, expressions attributives aux constructions sérielles et causatives-résultatives en passant par les 'techniques' inverses de transitivisation et d'intransitivisation ( $c f$. H. Seiler \& W. Premper,eds. 1991).

${ }^{8}$ On se reportera à l'ouvrage de Bybee (1985) pour l'agencement des morphèmes dans le mot.

${ }^{9} \mathrm{Il}$ n'y a pas de morphème de futur dans la conjugaison du finnois.

${ }^{10}$ Les abréviations des gloses sont les suivantes : A/M, auxiliaire/modalité (indonésien); \pm ANT, (antérieur) passé/présent ; $1 \mathrm{SG}, 1^{\text {ère }}$ personne du singulier ; ABES, abessif; ACC, accusatif; ACCO, aspect accompli ; ELA, élatif; ESS, essif ; GEN, génitif ; ILL, illatif ; INES, inessif ; INF1, premier infinitif (4 infinitifs) ; NOM, nominatif ; PART, partitif ; PASS, passif; PL, pluriel ; POT, potentiel ; PPA, participe passé ; PPR, participe présent ; PRES, présent ; PRET, prétérit ; PROSP, aspect prospectif ; SG, singulier ; T-Adv, adverbe de temps. ${ }^{11}$ Olson (1981), Van Valin et La Polla (1997), Klingler (2003).

${ }^{12} c f$. l'article de C. Noyau dans ce volume.

${ }^{13}$ L'indonésien, sous ses deux variantes dialectales : le malais de Malaisie et de Singapour et l'indonésien de l'archipel indonésien, est une langue de grande communication (plus de 250 millions de locuteurs) du groupe malayo-polynésien apparentée au tagalog des Philippines, mais qui présente une érosion grammaticale typique des koinê.

${ }^{14}$ Le préfixe me- est transformé par sandhi en meng-devant une occlusive sourde ou une voyelle.

${ }^{15}$ Les exemples (3-4) sont empruntés à Mintz 1994. Il est à noter qu'en l'absence de flexion verbale, le choix du temps dans la traduction française est piloté uniquement par la référence temporelle du TA.

${ }^{16}$ tadi signifie : il y a peu, il y a un instant, tout à l'heure (catégorie temporelle du révolu).

${ }^{17}$ Merci à Ph. Grangé de nous préciser que tolong désigne la forme nue, fréquente à l'oral, à laquelle se substitue menolong dans un niveau de langue standard ou soutenu.

Nous remercions Didier Bottineau (CRISCO) pour le temps qu'il a consacré à vérifier les données et l'argumentation de cette section.

${ }^{19} \mathrm{Ex}$ : Igandetan elizara joaten da: 'Il va à l'église le dimanche' (générique).

${ }^{20} \mathrm{Ex}$ : Elizara joaten ari da: 'Il va à l'église' (maintenant).

${ }^{21} \mathrm{Ex}$ : Galdu zen : 'Il se perdit'.

${ }^{22}$ Ex : Galdua zen : 'Il était perdu, il fut perdu, il s'était perdu'. 


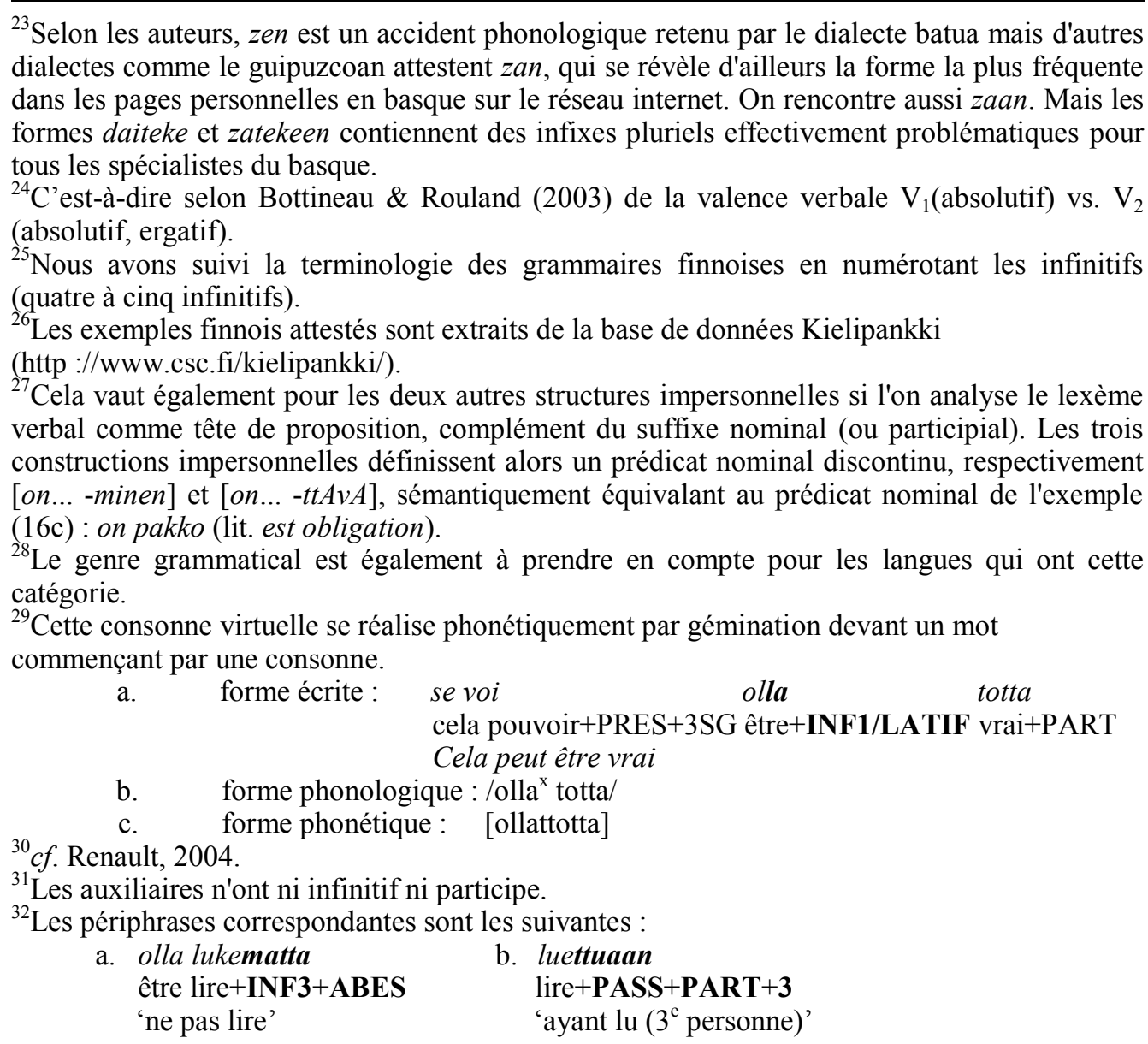

